\title{
Research on Detecting Thickness of High Temperature Float Glass by Laser Trigonometry Measurement of CCD Sensor ${ }^{\star}$
}

\author{
Wei Wang*, Zhaoba Wang \\ School of Information and Communication Engineering, North University of China \\ Taiyuan 030051, China
}

\begin{abstract}
It is very important to use the detection technology of float glass thickness in glass production. In the process of detecting glass thickness on line, we adopt laser trigonometry displacement detection theory. Directing at detection system theory in the high-temperature state, the paper does a further analysis. The paper analyzes the theory and experimental results by using the CDD detection method. The research improves the precision and stability of the detection, increases the glass production quality and cuts down the energy consumption and production cost.
\end{abstract}

Keywords: CCD; Trigonometry; High Temperature; Detection

\section{Introduction}

Generally, high temperature objects can show many different states, such as: solid state, liquid state, gaseous and plasma state. There are two kinds of methods to implement high temperature detection: one is Touch detection; the other is Un-touch detection. The former uses sensors to touch the detected object, whose method is characterized by high accuracy, easy operation and flexible use. But the location of detection is fixed, moreover, component of the sensor is easy to be destroyed. Therefore, the method causes an effect on the high temperature field. On the other hand, because the latter needn't touch the detected object, it doesn't destroy the temperature field of the object and can't ruin the component of the sensor. In view of these, the detection method causes less influence on production on line, so un-touch detection is a new trend of high temperature detection.

Glass thickness is not only a vital parameter in the process of glass manufacture, but also a crucial index in detecting quality of glass products. If the thickness of glass band is not stable,

\footnotetext{
${ }^{\star}$ Project supported by Shanxi Provincial Young Science Foundation (No. 2013021015-2) and the National Nature Science Foundation of China (No. 60672005)

${ }^{*}$ Corresponding author.

Email address: 41695559@qq.com (Wei Wang).
} 
it results in a lot of waste. However, we can detect the glass thickness in the hot side of the tinbath or annealing kiln, what's more, the research data can also be provided in time. In the way, time can be shortened and cost can be reduced and saved. Because environment temperature is about $600{ }^{\circ} \mathrm{C}$ in the hot side of annealing kiln, using the normal method to detect thickness is impossible.

\section{Detecting Principle}

The system employs Japanese Keyence LK-085CCD displacement sensor, which is a long-distance untouched displacement sensor and is acted as a light receiving component. Reflected lights from subject are focused on CCD through receiver lens group. The CCD determinates optical center by total optical distribution which enters light component. Moreover, it also adopts 32 super highspeed RISC processors to conduct signal processing. Then, take it as location of subject. However, because light distribution is influenced by surface condition of subject, it causes measurement errors. Its measurement reference distance is $80 \mathrm{~mm}$, measurement range is $15 \mathrm{~mm}$, light spot diameter of reference distance is $70 \mu \mathrm{m}$, resolving capability is $3 \mu \mathrm{m}$, sampling period is $1024 \mu \mathrm{s}$, analogy output is $5 \mathrm{~V}(3 \mathrm{~mm} / \mathrm{V})$, then displacement voltage by its controller LK-2110 can be obtained. Its principle picture is listed in Fig. 1.

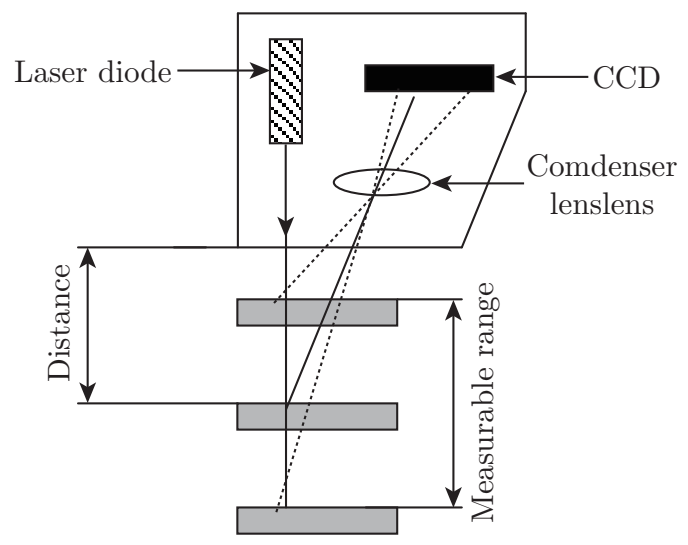

Fig. 1: CCD sensor measurement principle picture

Fig. 2 shows the signal waves which are formed on the surface of standard echo object (white paper) and $2 \mathrm{~mm}$ white glass by the laser trigonometry displacement sensor. The differentiating power of sensor is $5 \mu \mathrm{m}$ when the system detects the standard object. It can show that the echo signal of the glass surface has low delicacy and high noise. Thus, the data need to be dealt with to meet the demand of the detection with a special method.

The research should be implemented to increase the signal amplified times and special signal disposal technique. So we meet the detection demand by means of sacrificing differentiating power (less than $20 \mu \mathrm{m}$ ).

In Fig. 3, it can display the detection project of glass thickness. We adopt two CCD laser displacement sensors, which are installed in the upside and downside of glass band to simultaneously detect the displacement of the upside surface and downside surface of glass band. We can calculate the glass thickness based on the displacement of the two sensors. In the practical process of production, float glass is passed on transferring roller. Inevitably, there exists jitter and 


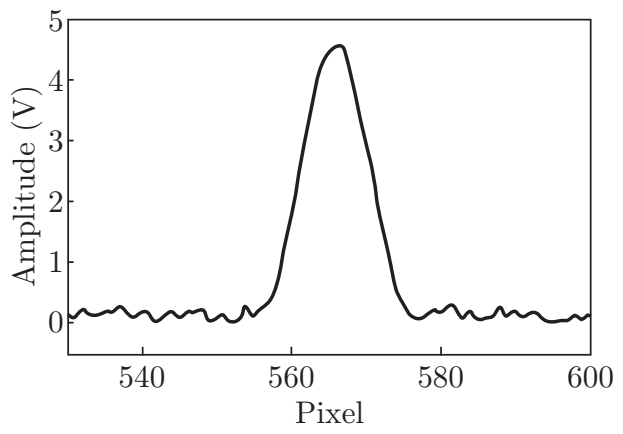

(a) Standard object

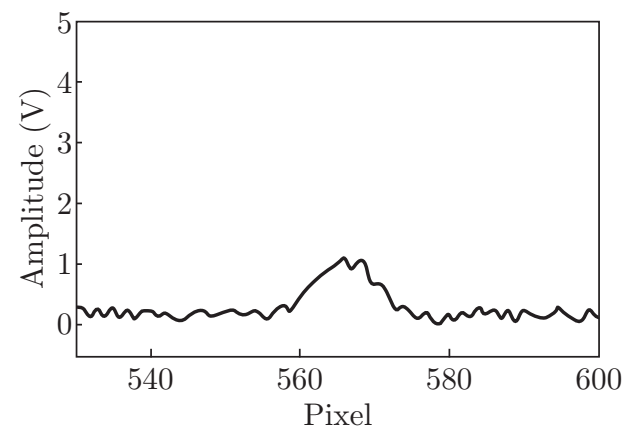

(b) Glass surface echo

Fig. 2: Sensor output from difference objects

tilt. Therefore, we symmetrically place two sets of laser sensors, which are efficient to eliminate measurement errors. Glass is a kind of transparent object, so in the measurement process, in order to avoid that interference wave from one sensor may be brought to the other one, we stagger several mms distance between two CCD sensors in vertical direction of measurement location.

During the practical measurement process of using CCD sensor, appropriately installing two sets of displacement sensors at a slant can make a quantity of lights of CCD enlarged and increase sensor resolution. But because of tilt installation from sensor, light is incident. It causes that there is an angular relation between voltage output value which controller LK-210 gathers and factual distance value. It is shown as follows in Fig. 4.

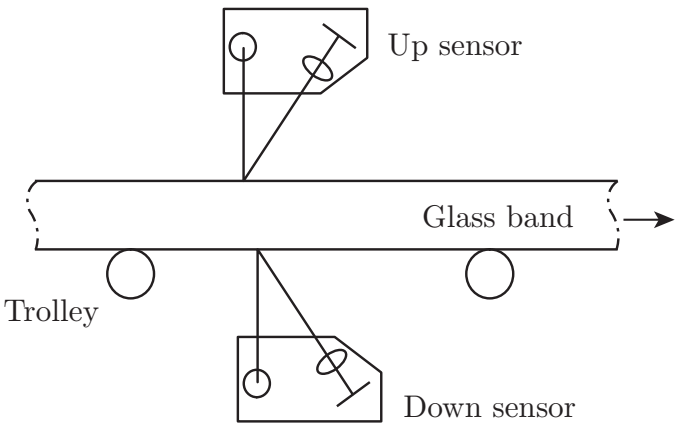

Fig. 3: Detection method of glass

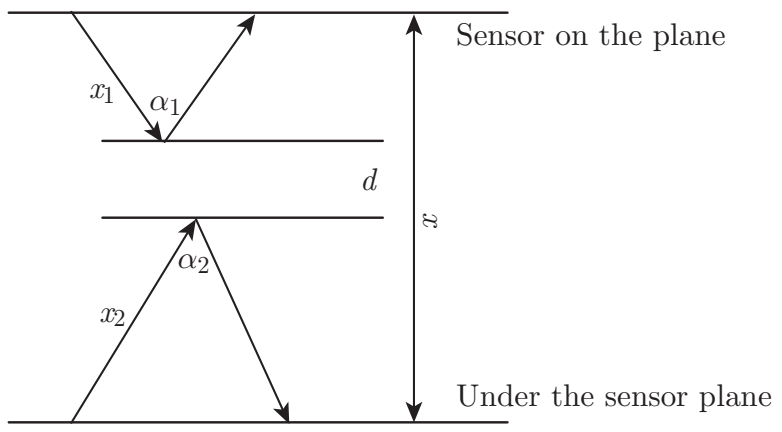

Fig. 4: Signal reflection principle

From triangular relationship in the picture, the calculation formula is:

$$
d=x-x_{1} \cos \alpha_{1}-x_{2} \cos \alpha_{2}
$$

In Eq. (1), we use gauge block to demarcate relevant parameters, such as: $\cos \alpha_{1}, x_{2} \cos \alpha_{2}$ and $x$.

\section{Detection Data}

Within the detection range of the two sensors, 22 detection data are obtained which are used to detect position, and the distance is $1 \mathrm{~mm}$ between every two detection positions.

We arrange three groups of sensors in the practical detection system. Every group has two sensors, one is located over the glass and the other is located under the glass. One group is in 
the middle of the glass band, and the rest of two groups are in the two sides of the glass band. In the system the detection data can be gotten, which are listed in Table 1.

Table 1: Detection data

\begin{tabular}{cccc}
\hline Detection position & Detection data $(\mathrm{mm})$ & Detection position & Detection data $(\mathrm{mm})$ \\
\hline 1 & 3.384 & 12 & 3.430 \\
2 & 3.413 & 13 & 3.432 \\
3 & 3.431 & 14 & 3.435 \\
4 & 3.437 & 15 & 3.433 \\
5 & 3.437 & 16 & 3.431 \\
6 & 3.438 & 17 & 3.435 \\
7 & 3.436 & 18 & 3.438 \\
8 & 3.435 & 19 & 3.436 \\
9 & 3.433 & 20 & 3.432 \\
10 & 3.432 & 21 & 3.419 \\
11 & 3.430 & 22 & 3.395 \\
\hline
\end{tabular}

Fig. 5 is the result of the data analysis by the matlab.

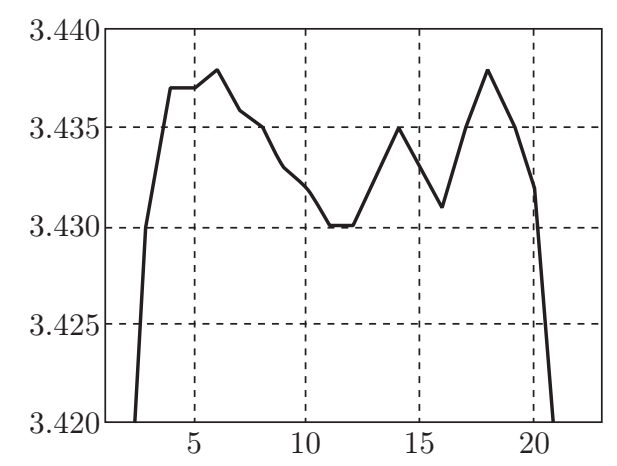

Fig. 5: Data analysis

The thickness error is controlled within $0.02 \mathrm{~mm}$ in the twenty detection positions from the result of Fig. 5. The detection not only covers the different glass thickness within the country standard, but also the detection precision is higher than the country standard.

\section{Heat Distribution and Optical Features Research on High Temperature Float Glass}

There is a basic hypothesis about laser triangulation method, i.e., air index of refraction "n" is a uniform distribution and " $\mathrm{n}$ " is a constant, otherwise measurement precision will be influenced. In the practical measurement environment, air index of refraction isn't a constant, but it varies in certain range. In general temperature and humidity, air index of refraction will range from 1.00022 to 1.00046 . 
In CCD sensor triangulation method, if index of refraction distributes uniformly, measurement precision will not be influenced. Once air index of refraction has non-uniform distribution, light transmission can be curved and has an effect on precision. Thus, we need do a further research on temperature of measurement structure.

At first, let's make a discussion about air temperature distribution of high-temperature glass band on line. According to thermodynamics principle, suppose that float glass band is enough big and consider that vicinity of glass band is a one-dimensional air wall. If air reaches to stable temperature, it is not endothermic and exothermic any more. Air temperature distribution is shown in following formulas:

$$
\begin{aligned}
t(x) & =\frac{1}{\beta k_{0}} \sqrt{k_{1}^{2}+\left(k_{2}^{2}-k_{1}^{2}\right) x / L}-\frac{1}{\beta} \\
k(t) & =k_{0}(1+\beta t)
\end{aligned}
$$

In Eq. (2) and (3), $x$ represents distance scope of glass band; $k$ is an air conductivity modulus which varies with temperature; $\beta$ is a temperature modulus. Suppose $t_{1}$ is temperature of glass band, $t_{2}$ is the air temperature of place $L, k_{0}, k_{1}$ and $k_{2}$ respectively represent modulus of air conductivity when it reaches $0{ }^{\circ} \mathrm{C}, \beta=0.0018182, k_{0}=0.002567, t_{2}=30{ }^{\circ} \mathrm{C}$.

When $\mathrm{t} 1$ is $50{ }^{\circ} \mathrm{C}$, put it into the formula mentioned above and get the data, similarly, when $\mathrm{t} 1$ is $100{ }^{\circ} \mathrm{C}$, t1 is $200{ }^{\circ} \mathrm{C}$, $\mathrm{t} 1$ is $500{ }^{\circ} \mathrm{C}$, we get Fig. 6, which describes temperature distribution in the neighbourhood of glass band.



Fig. 6: Temperature distribution of glass band vicinity

These factors, such as: absolute temperature $T=t+273.15$, air pressure $P$, humidity $e$, wave length $\lambda$ and other parameter conditions, can influence the air index of refraction.

$$
\begin{aligned}
& n=1+N(\lambda, P, T, e) * 10^{-6} \\
& N(\lambda, P, T, e)=7.7748^{\frac{P}{T}}(1+0.006063 \lambda-2)+0.1127^{\frac{e}{T}}
\end{aligned}
$$

When $\lambda=0.65 \mu \mathrm{m}$, atmospheric pressure $P=101325 \mathrm{~Pa}$, humidity $e=0$, according to Eq. (4), $n(T)=1+0.0797663 / T$ can be obtained. It can be found that index of refraction and absolute 
temperature have relationship. Eq. (2) and (3) can get air reflective index distribution data in the neighourhood of hot glass band. Temperature are $50{ }^{\circ} \mathrm{C}, 100{ }^{\circ} \mathrm{C}, 200{ }^{\circ} \mathrm{C}, 500{ }^{\circ} \mathrm{C}$ respectively as shown in Fig. 7.

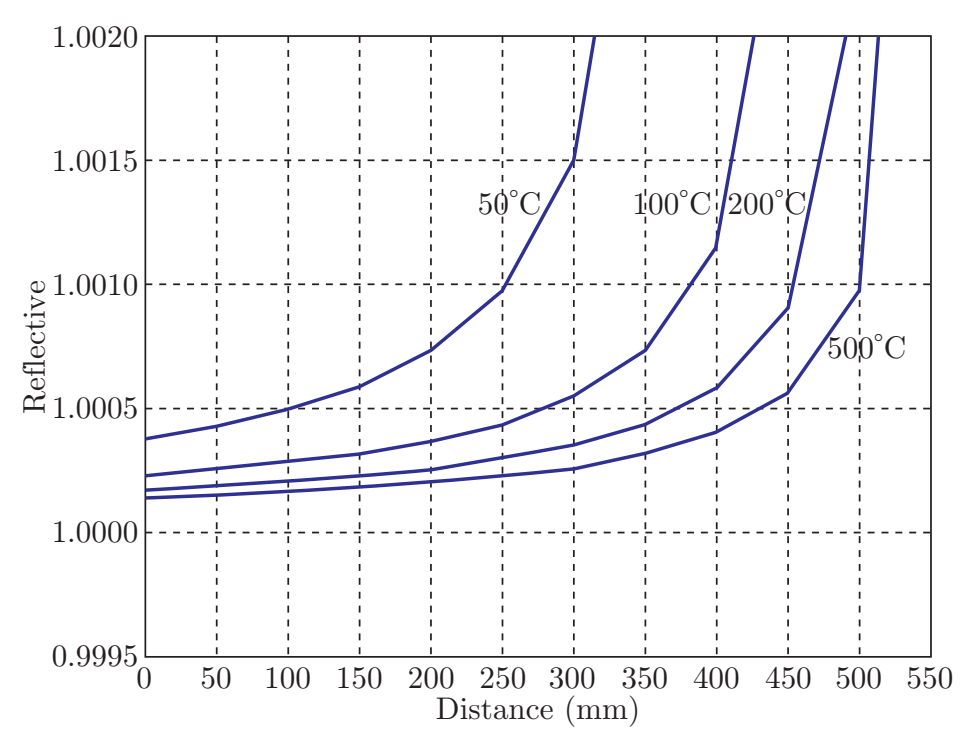

Fig. 7: Air reflective index distribution of glass band vicinity

\section{$5 \quad$ High Temperature Thickness Measurement Model}

On the basis of analyzing temperature distribution in the vicinity of glass band and the air index of refraction, if the research wants to get glass measurement features in high temperature state, we need to further analyze glass thickness model. According to the model, let make an analysis about the precision of sensor and stability of data-gathering.

Refraction of light formula is:

$$
n \sin \theta=n^{\prime} \sin \theta^{\prime}
$$

In the formula, $n$ and $n^{\prime}$ represent the incident and emergent of refractive index respectively, $\theta$ and $\theta^{\prime}$ stand for the incident angle and the emergent angle respectively. If light is in the uniform medium, $n$ and $n^{\prime}$ are always equal, $\theta$ and $\theta^{\prime}$ are also always equal, thus light transmits through straight line. In the practical measurement process, $n$ and $n^{\prime}$ vary with spatial location, $\theta$ and $\theta^{\prime}$ also vary with spatial location, therefore light will be curved. The bigger incidental angle $\theta$ is, the bigger change of $\theta^{\prime}$ is. In the situation of the same vertical distance, if journey of light is longer, the deviation of light is more serious as shown in Fig. 8.

According to Eq. (6), Eq. (7) is,

$$
\sin \theta(x)=n_{0} \sin \theta_{0} / n(x)
$$

In the formula, $n_{0}$ and $\theta_{0}$ are refractive index of air and light emission angle respectively when $x=0$. Thus, according to angle relationship, displacement formula can be deduced in vertical direction:

$$
y(x)=\int_{0}^{x} \operatorname{tg} \theta(x) d x=\int_{0}^{x} \sin \theta(x)\left(1-\sin ^{2} \theta(x)\right)^{-\frac{1}{2}} d x
$$




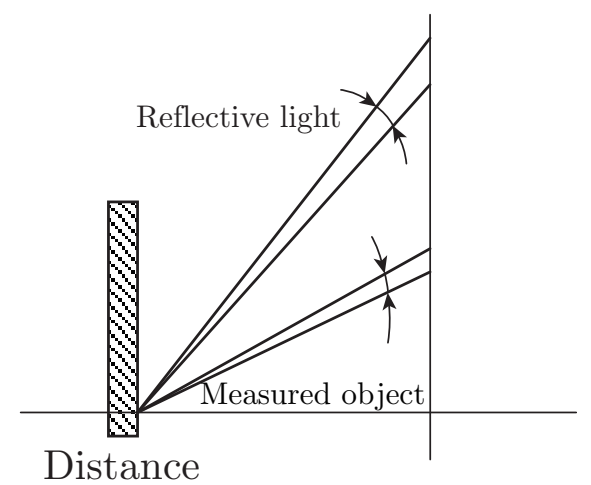

Fig. 8: The relationship between light deviation and angle

According to CCD sensor installation angle, let take value of out shooting angle as $12.5^{\circ}$ and combine with Eq. (7) and (8) to analyze data, then location deviation is obtained in Fig. 6 when temperature reaches to $50{ }^{\circ} \mathrm{C}$.

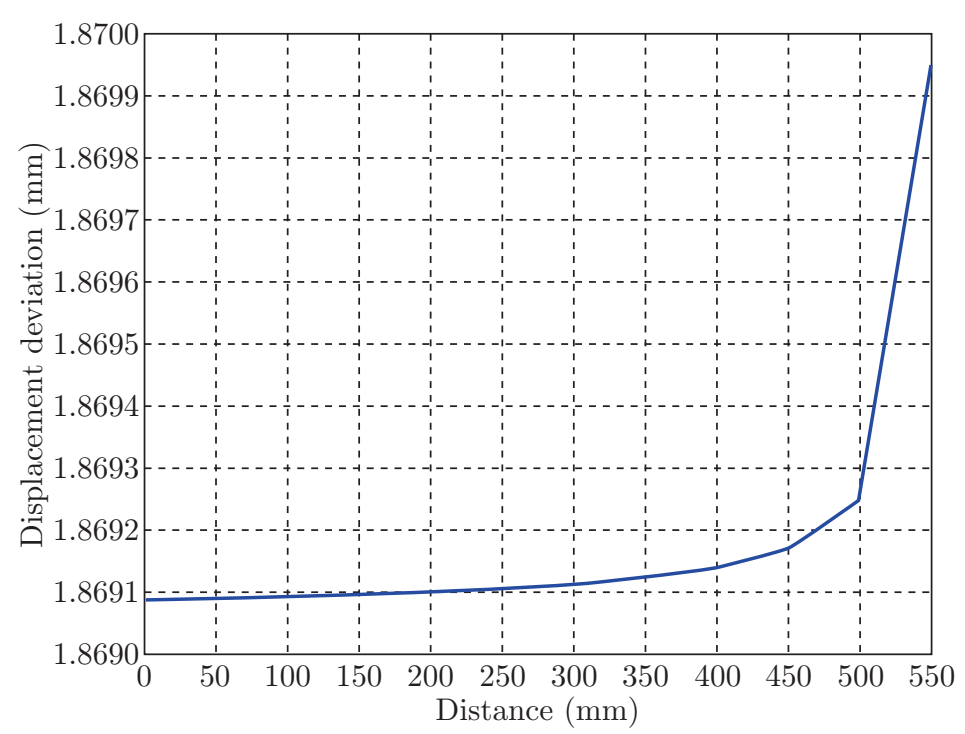

Fig. 9: Displacement deviation with change of measured place

On the basis of measurement location and temperature change, the change trend of measurement deviation can be gained. According to glass measurement features, we get glass thickness change model under the condition of relevant high temperature. Take advantage of on-line high temperature thickness model to analyze data that sensors gather. It can improve precision and stability of on-line measurement.

\section{Conclusions}

Through the study of float glass thickness detection technology in high-temperature state, the paper establishes a real-time thickness detection system based on CCD displacement sensor. Conducting relevant theory research and experimental analysis on high temperature float glass thickness CCD measurement, we also do a further study on system measurement principle, de- 
tection method in the state of high temperature, refractive index of light, thickness measurement deviation. The research has higher practical value and brings a bright prospect. The technology also offers an efficient way for on-line detection of float glass thickness.

\section{References}

[1] H. Yükselici, P. D. Persans, High temperature optical properties of CdS crystals in borosilicate glass [J], Journal of Non-Crystalline Solids, 203 (1996), 206-210

[2] A. B. Shelekhin, S. Pien, Y. H. Ma, Permeability, surface area, pore volume and pore size of Vycor glass membrane heat-treated at high temperatures [J], Journal of Membrane Science, 103 (1995), 39-43

[3] S. Inaba, S. Oda, K. Morinaga, Heat capacity of oxide glasses at high temperature region [J], Journal of Non-Crystalline Solids, 325 (2003), 258-266

[4] Daniel Isabelle, McMillan Paul F., Gillet Philippe, Poe Brent T., Raman spectroscopic study of structural changes in calcium aluminate $(\mathrm{CaAl2O} 4)$ glass at high pressure and high temperature [J], Chemical Geology, 128 (1996), 5-15

[5] Sung-bum Sohn, Se-young Choi, Gyeung-ho Kim, Hue-sup Song, Goo-dae Kim, Stable sealing glass for planar solid oxide fuel cell [J], Journal of Non-Crystalline Solids 2002, 103-112

[6] Z. L. Kahn-Jetter et al., Three-dimensional displace ment measurements using digital image correlation and photo grammetric [J], Exp. Mech., Vol. 30, No. 2, 1990, 10-16

[7] Rosanne M. Guijt, James P. Armstrong, Esme Candish, Veronica Lefleur, William J. Percey, Microfluidic chips for capillary electrophoresis with integrated electrodes for capacitively coupled conductivity detection based on printed circuit board technology [J], Sensors and Actuators B: Chemical, Volume 159, Issue 1, 28 November 2011, 307-313

[8] P. Castellini, L. Stroppa, N. Paone, Laser sheet scattered light method for industrial measurement of thickness residual stress distribution in flat tempered glass [J], Optics and Lasers in Engineering, Volume 50, Issue 5, May 2012, 787-795

[9] D. Maki, T. Nagai, F. Sato, Y. Kato, T. Yamamoto, T. Iida, Microscopic dose measurement with thin radiophotoluminescence glass plate [J], Radiation Measurements, Volume 46, Issue 12, December 2011, 1543-1546 